\title{
E DI TORI AL
}

Apubli cação deste númer 0 marca o i ní ci o das comemor ações dos set ent a anos da Rodriguésia, o que acreditamos ser moti vo de júbil o para toda comunidade botânica brasil eira. Ao longo destas sete décadas, a Rodri guési a vempubl i cando i mortantes contri bui ções par a o conheci ment o botâni co e com mui to empenho consegui u superar as di fi cul dades que af etama cont i nui dade das publ i cações ci entíficas no paí s. Neste mmento de comemoração, nada mai s oport uno que a i mplement ação de al terações vi sando o mel hor cumpri mento de sua mi ssão, que é promver a di fusão ci entífica de qual i dade emtodas as áreas da bi ol ogi a vegetal. A partir deste vol ume a revi sta conta emseu Corpo Edi torial com sete editores de área, pertencentes a di ferentes instituições no Brasil e no exterior. Coma nova estrut ura procuramos garantir a mel hor assessori a possí vel para os trabal hos submet i dos. Nos úl ti mos anos, o número de manuscri tos encami nhados temaument ado expressi vamente, perm ti ndo que a partir deste vol ume sej ampublicados três números anuais. Os recentes avanços na estrut uração interna têmpermitido mai or rapi dez nas etapas de aval i ação e na publ i cação dos artigos. Al ém di st o, têmpossi bil i tado a obt enção de recursos fi nancei ros para a publ i cação da revi sta, incl ui ndo arti gos commai s de 30 I audas, semcustos aos autores e comacesso l i vre aos trabal hos publ i cados no si te do J BRJ.

Como marco desta ocasi ão especi al, este númer o é i nt ei ramente dedi cado à publ i cação de trabal hos sobre a flora da Reserva Florestal Adol fo Ducke, área com $100 \mathrm{~km}^{2}$ de floresta de terra firme, I ocal i zada em Manaus. Trata-se da pri mei ra fl or a publ i cada para a Amazôni a brasileira desde a Flora brasiliensis (1840-1906), a qual contou compouco material desta regi ão. Os I evant ament os fl orí st i cos na Amazôni a i nt ensi ficaram-se somente no sécul o XX, parti cul ar mente como própri o Adol fo Ducke. As pri mei ras i déi as sobre el aborar uma fl or a na Reserva Ducke foraml evant adas na década de 1960 por Marl ene Freit as da Si I va, W II i am Ant ôni o Rodri gues e Ghi II ean T. Prance. 0 proj et o ganhou fôl ego na década de 1990, quando for amest abel eci dos seus princi pai s obj eti vos, representados pel a el aboração de umchecklist, do tratamento taxonômi co para as faḿl i as e de umgui a prático de identificação. 0 check-l ist já se encontra di sponí vel emmei o el et rôni co e umgui a de i dent i ficação at é o ní vel de espéci e amplamente il ustrado foi publ i cado em 1999. Os trat ament os taxonôm cos passam a ser publicados na Rodriguési a a partir deste númer 0 , sendo que o próxi mo dedi cado a este proj et 0 está pl anej ado para 0 ano de 2006. Nest e pri mei ro número apresentams um artigo introdut óri 0 abordando a hi st óri a do Proj et 0 , caracteri zação da área da Reserva Ducke, I evantament 0 de probl emas e possí vei s sol uções para o estudo da fl ora amazôni ca, segui do de 35 monografias que tratam de vi nte famíl i as de pteridófitas e qui nze famíli as de angi ospermas, total i zando 93 gêneros e 165 táxons.

A publ i cação deste número só foi possí vel graças à i nesti mável dedi cação de Mi ke Hopki ns ( UFRA) e Cynt hi a Sother s (RBG Kew). Cont ou ai nda coma val i osa cont ri bui ção dos segui ntes pesqui sador es na revi são dos arti gos: Al essandro Rapi ni (UEFS); Andréa Cost a (UFRJ); Cl audi ne Massi Mynssen (J BRJ) ; El si e Gui marães (J BRJ) ; J ef f er son Prado (I Bt-SP); Lana Syl vest re (UFRRJ); Lúci a Lohmann (USP); Marl i Pi res Mori m(JBRJ); Mi I t on Gr oppo (I Bt-SP); Regi na Andreat a (USU) e Vidal Mansano (J BRJ), para os quai s expressamos nossos agradeci ment os.

Leandro Freit tas

Gest or do Corpo Edi torial
Raf ael a Campostrini Forzza

Edi tora-chefe 